\title{
Performance Improvement in Steam Turbine in Thermal Power Plants Using Artificial Neural Network
}

\author{
Fawaz S. Abdullah*, Ali N. Hamoodi, Rasha A. Mohammed \\ Department of Power Techniques Engineering, Faculty of Technical Engineering Collage, Northern Technical University, \\ Mosul 41001, Iraq
}

Corresponding Author Email: Fawaz.sultan@ntu.edu.iq

https://doi.org/10.18280/jesa.540611

Received: 10 August 2021

Accepted: 25 September 2021

\section{Keywords:}

steam turbine governor, performance, PID

controller, ANN controller, MATLAB

modeling

\begin{abstract}
Artificial intelligence has proven its effectiveness in many industrial fields to enhance the existing functionality. Artificial intelligence and machine learning algorithms integrated with turbines can be useful in controlling important variables such as pressure, temperature, speed, and humidity. In this research, the Simulink library from MATLAB is used to build an artificial neural network. The NARMA L2 neural controller is used to generate data and for training networks. To obtain the result and compare it with the realtime power plant, data is collected. The input variables provided to the neural network have a large effect on the hidden layer and the output of the neural network. The circuit board used in this research has a DC bridge, a transformer and voltage regulators. The result comparison shows that the integration of artificial neural networks and electric circuits shows enhanced performance with high accuracy of prediction. It was observed that the ANN integration system and electric circuit design have a result deviation of less than $1 \%$. This shows that the integration of ANN improves the performance of turbines.
\end{abstract}

\section{INTRODUCTION}

A steam turbine is a tool which extract thermal energy from pressure of steam and convert into an energy for performing mechanical work. It is mostly used in the generation of electric power in power plant. A steam turbine is consisting of high pressure, low pressure and moderate pressure. The highpressure part of steam turbine contains two casing which split horizontally. Inner split is fixed inside and it is present in the axial direction, and an outer casing which can provide an expansion possibility in every direction.

The Intermediate-pressure part of steam turbine contains three parts which split horizontally. These three parts an attached vertically using flanges. Outer branch is rigidly attached with condensers using spring. At the inner casing of the intermediate pressure, low pressure heater's tube nest is placed. At the centre of low-pressure casing part, in the axial direction, guide key and fix point are connected with the casing [1].

An artificial neural network is highly adapted to the improvement of the workings of a steam turbine. In the proposed research, the need for fast prediction to control the power engineering operation was mentioned, and by using the inverse neural network control algorithm, it was addressed. This technique adjusts the temperature in the steam turbine to maintain it below the threshold value for safety, and at the same time, it maintains the start-up rate [2]. Salim et al. [3] used design and implantation of electric circuits for enhanced performance of steam power plants and artificial neural networks as techniques. The novelty in this paper was in applying the NARMA controller for the purpose of enhancing turbine performance. Comparing the results from modelling by ANN and electric circuit with experimental data reveals a good agreement and the maximum deviation between the experimental data and predicted results from ANN and circuit design is less than $1 \%$.

Various researches have proposed genetic algorithms, differential evolution technique and different artificial neural network technique to improve the performance of steam turbine [4].

In turbine algorithm, controller implementation is carried out using the neural network. In many industrial application, neural network is used to control the stress in steam turbines [4]. Dominiczak et al. [5] proposed a mathematical model Nonlinear Auto-Regressive neural networks with exogenous inputs (NARX) for maintaining the stress of steam turbines.

Dettori et al. [6] proposed Nonlinear Model Predictive Control (NMPC) technique to improve the sped of steam turbine start-up and increases the energy production with maintaining the stress of steam turbines.

Based on the above survey and although the pressure control neural network of steam turbines has been used in many industrial applications, the use of the adaptive fuzzy neural inference model is limited. Therefore, for generating the turbine cycle output, adaptive neuro-fuzzy inference model is used in this research. Input of the adaptive neuro-fuzzy model was rate of waterflow, its temperature, it's pressure and pressure at condenser at each cycle. The result of this research suggest that the underlying system predicts the output with high accuracy and it compute the output of generator correctly.

The aim of the proposed research is to improve the power plant performance by integration the artificial neural network technique by maintaining different parameters like velocity, temperature, pressure and humidity. 


\section{ARTIFICIAL NEURAL NETWORKS}

ANN works similar to the human brain with many nerves. There exist multiple layers of nodes which connect to form a network. It was first introduced in the 1940 by Mc Culloch. There exist multiple tools and types of artificial neural network. The model of artificial neural network is created based on the type and an input which is provided to it. It is useful in solving the statically difficult problem and it generally provides an output with some probability distribution which suggest the prediction based on the input values. Neural network is an interconnected network of multiple nodes refereed as neurons. Model formed by neural network is an algorithmic pattern which can process any input.

Artificial neural network is generated in two steps, first one is training and second is testing phase. In training phase, an input is provided to a neural network, this is usually a big data and hence a pre-processing of data is required before it feed to the neural network. In the training phase, neural network algorithm creates an artificial intelligent model consist of several iteration. After the model formation, neural network model can be used for a prediction purpose. However, to test the accuracy of the network, testing phase is required. In testing phase, a split technique is used to split the whole data into a bag of data and other dataset is used as a training data and the accuracy of the model is determined using remaining dataset. Depending on the accuracy of the model it is used for the predication or decision-making purpose [7-10].

The Artificial neural network is a technique of Artificial Intelligent to create an AI model for predication from the external data. Multiple layers present in the artificial neural network is useful in improving the accuracy when compare to other artificial intelligence algorithm like classification and clustering technique. Figure 1, illustrates the architecture of artificial neural network. Feed forward neural network is used to generate a multi-layer neural network. In the multi-layered network, output of previous layer is input to the next layer. Backpropagation is a method of adapting the weight of network using the error rate of the previous layer. An error rate is calculated by obtained and desired output value. Backpropagation is a technique to handle or adapt the missing attributes from the training data [11].

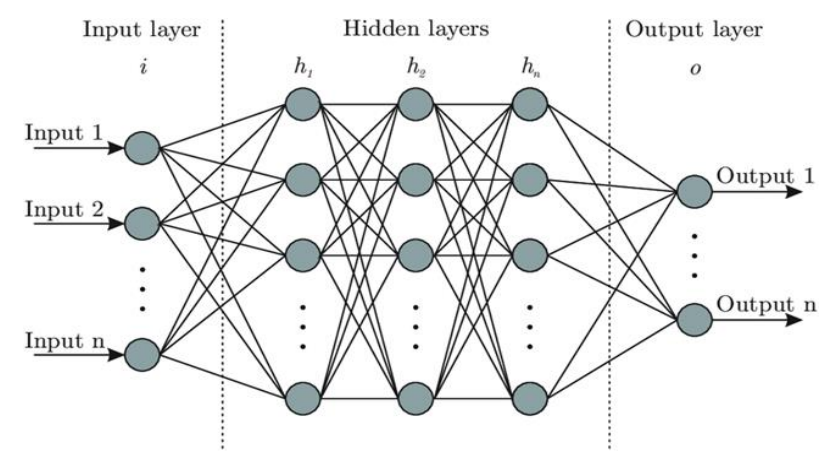

Figure 1. Architecture of Artificial Neural Network

\section{DATA COLLECTION AND PROPOSED METHOD}

A steam turbine is mostly used in the power plants as it is more cost efficient if compared with other peer with respect to performance and capacity of power plant. For increasing the thermal capacity of the steam turbine various complexity levels are available. Using the artificial intelligent technique, the complexity of steam turbine can be studied and analysed which considers the high, medium and low-pressure stage of steam turbine. Using the model prepared by artificial neural network can be used for designing, synthesis, generating simulation and to monitor the power plant control system [1217]. 160-Megawatt steam power plant has different motives like steam extraction, heater for feeding water and separator for moisture. MATLAB software is used to simulate the different factors which affects the working of AL-Dura power plant steam turbine, this includes pressure at steam turbine, its temperature, current humidity and velocity. This simulation is based on the data which is obtained from the real power plant.

\subsection{Data collection}

The input variable which affects the working or operating of power plant is as follow:

(1) Steam turbine pressure

As the data received from the real time power plant, a pressure which is applied for the safety operation at steam turbine has to be less than 140 bar.

(2) Temperature

As the data received from the real time power plant, a temperature which is applied for the safety operation with avoiding any damage to turbine blades at power plant has to be less than $550^{\circ}$ Celsius.

(3) Humidity

Humidity is an important factor which affect the blades which rotates in steam turbine. A humidity at steam turbine has to be less than 0.12 .

(4) Velocity

Speed or velocity of the turbine is also an important factor in the power plant as it affects mechanical power which is responsible for efficient operation. The ideal velocity of the steam turbine should be greater than 3500 rotation per minute.

From the collected data input variable and output parameter is used for building an artificial neural network. Raw Data is collected for 15 days from the sensor inputs and it is consisting of 64500 records. As described in the previous section, from whole collection of data half is used for training the artificial neural network and other is used for testing purpose.

An experimental device is consisting of hardware and software. This includes a laptop with Microsoft operating system, input output user, interfaces and controllers. The device is integrated with multiple sensors like temperature, humidity capturing sensor, sensor for detecting velocity and pressure. Built in sensors are capable to detect the sensor response and it send the signal to interfaces. As the data collection is performed using sensor inputs, it contains a periodic noise. In this research Fourier transformation is used for noise removal. An interface is responsible to send the signals to the operating system and the software which is installed in the operating system. This software provides an output for selection to operators. Sensors are consisting of DC bridge and electric transformers. DC bridge is responsible for conversion of signal into the continuous voltage signal in the digital form. In this zero's and one's representing zero volt and five volts respectively. Whereas electric transformers maintain the current and voltage at the supply. Table 1 shows system information.

Voltage regulator is used for regulation purpose and voltage signal is send to it. Voltage regulator is responsible for stabilizing the voltage and it only permit the output voltage to 
certain limits which is configured. If the sensor reading is high then the conversion of analog signal to digital signal takes place. Figure 2 describes the sensor diagram. In regards with software MATLAB program is used for the analysis and processing purpose.

Table 1. System information

\begin{tabular}{cc}
\hline Operating system & Windows 10 \\
\hline RAM & 8 GB DDR 4 \\
Processor & Intel Core i5 \\
Software & MATLAB \\
SW version & R2021a \\
\hline
\end{tabular}

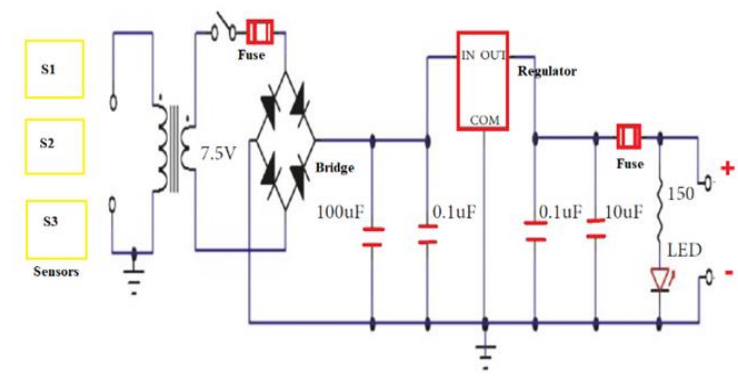

Figure 2. Sensor diagram

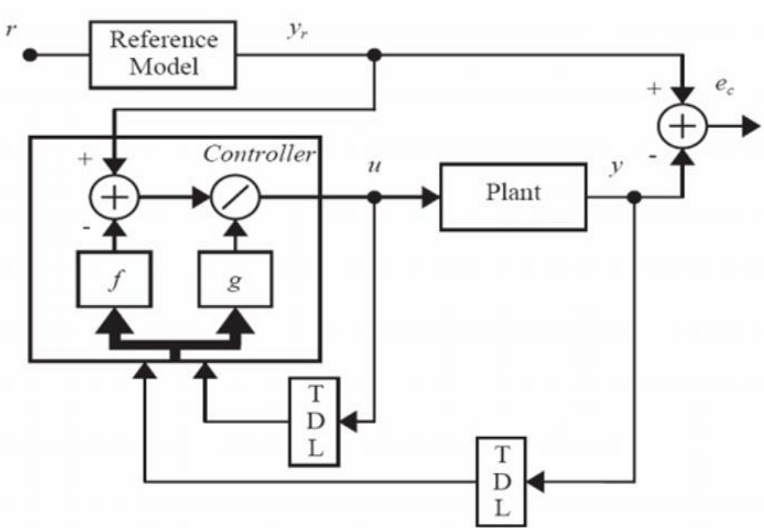

Figure 3. Block diagram of NARMA L-2

Non-linear regression moving average neural network is used in this research, which maintains the humidity, pressure at steam turbine, temperature and velocity of turbine. NARMA model is used in the artificial neural network for the simulation in MATLAB tool. The aim of this model is to adjust the magnet position which is present on the electromagnet and it rotates in the vertical direction. System with NARMA controller is illustrated in Figure 3.

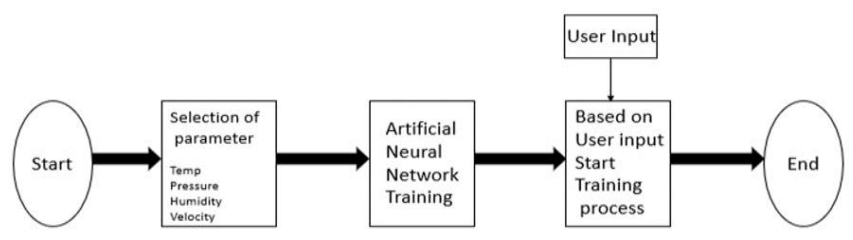

Figure 4. Flow chart of NARMA model

Figure 4 shows the flow chart of working of the NARMA model. In this the first step is to selection of identification parameter. In this the minimum and maximum interval is selected as 0.1 and 1 second respectively. In the next step data is imported and the plant's information like output and input is fetched from the NARMA. After the data collection process, actual artificial neural network model is generated based on collected data. Based on the response of the user, a training model is selected and the output is displayed on the User interface.

\section{RESULTS AND DISCUSSIONS}

Using the data collected from an experiment setup, the neural network turbine model is simulated in MATLAB software. Backpropagation technique of neural network creates various hidden layers in neural network and based on the error rate nodes on the hidden layers are either updated or added. It improves a precision accuracy of the model. For the accuracy comparison mean square error rate is used.

Figure 5 illustrates the performance chart of the training model with 2 hidden layers in artificial neural network. Performance chart shows that above 85 epoch a system generates an expected output with higher accuracy and stability in the result. In this setup 2 hidden layers are configured however in the NARMA every variable is controlled by 14 hidden layers. This increases the accuracy by minimising the error rate.

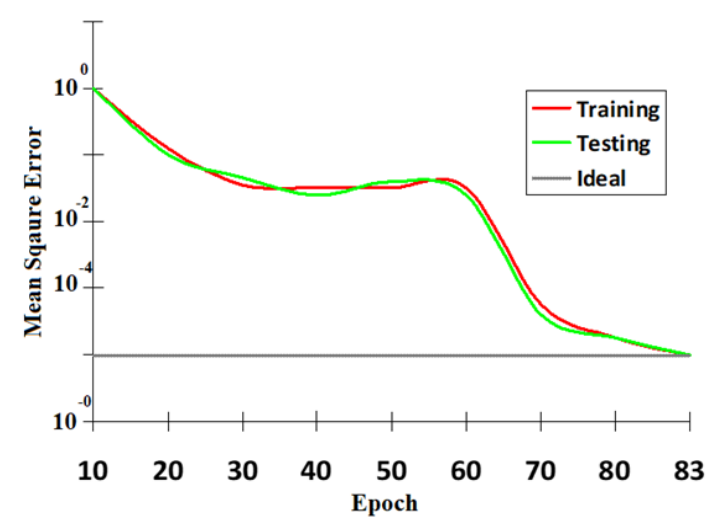

Figure 5. Performance of the training model

Simulink MATLAB model is used for simulation and analysing purpose, using it various input variables which affects AL-Dura power plant steam turbine is controlled. In the proposed model for every signal, neural network is created and then a centralised processing unit is used to analyse the overall output of each processing.

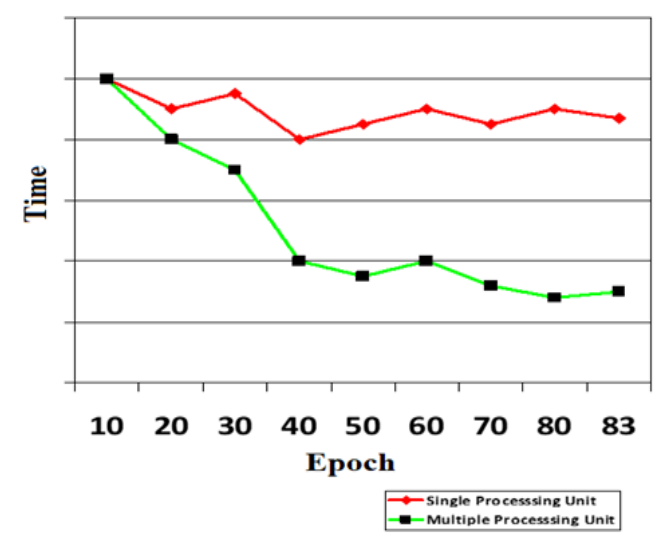

Figure 6. Time evaluation of proposed system 
This reduces the time required for processing and improves the time complexity of the system. Figure 6 shows the time complexity comparison of proposed system with single processing unit. The individual processing units are responsive of all input variables which are pressure, velocity, temperature and humidity.

As the neural network compromises of multiple hidden layers, it increases the training time, hence a parallel training approach is proposed. This also improves the steady state of the system as even one processing is down system remains up based on other processing units.

For the performance testing proposed neural network algorithm was compared with support vector machine algorithm for the given inputs. It is observed that the performance of artificial neural network is better than support vector machine. Figure 7 shows the performance comparison of support vector machine and artificial neural network for the provided inputs.

Result analysis of the model shows that the temperature of steam turbine at power plant need to maintain at $550^{\circ}$ Celsius. Figure 8 (a) illustrates the temperature chart with considering the time offset. Result analysis of velocity shows that the ideal velocity for steam turbine rotation is $2500-3000$ rotation per minute. Figure 8 (b) illustrates the velocity analysis with respect to time offset. Result analysis of Humidity shows that the surrounding humidity of steam should be $0-0.12$. Figure 8 (c) illustrates the humidity analysis with respect to time offset. Results shows that the pressure which should be applied at steam turbine has to be 140 bars and towards condenser it gets further reduced to 6 bars. Figure 8 (d) illustrates the pressure analysis with respect to time offset.

This result shows the permissible levels for the pressure, humidity, velocity and temperature. It was observed that the
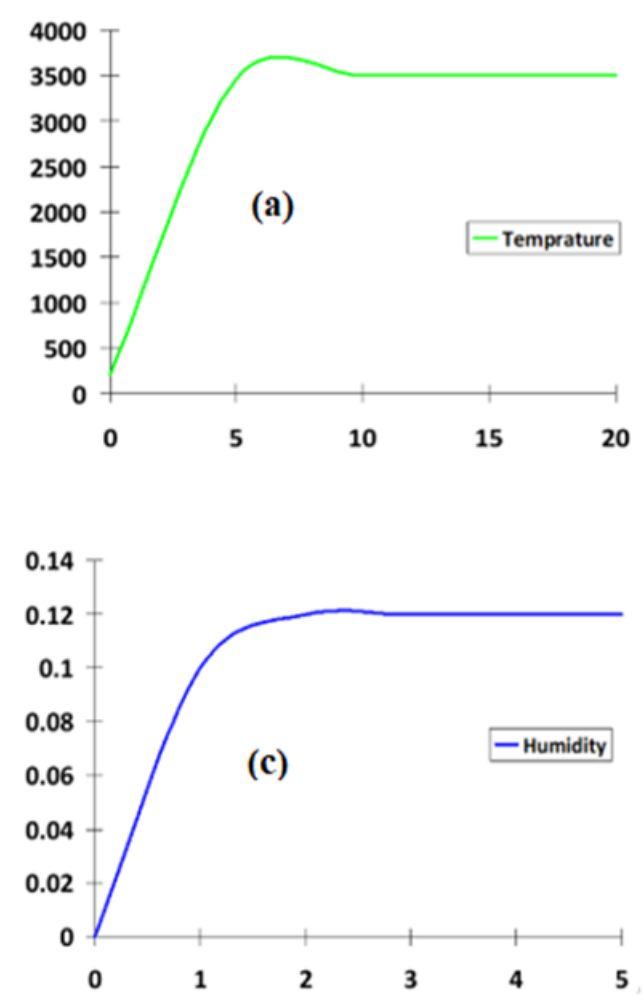

permissible temperature is $550^{\circ}$ Celsius but it was observed that the pressure is directly proportional to the temperature.

As pressure rises, there is an increment in temperature too. This limit of temperature avoids any type of melting or other heating issue in the power plant. Humidity captured from the device shows that permissible limit of humidity is 0.12 at which there is no humidity related risk.

It is also observed that the it is depended and inversely proportional to the temperature and pressure. As the temperature and pressure increases, the humidity decreases it avoid the erosion and corrosion of the turbine blades.

The comparison of humidity levels between the circuit design and artificial neural network is carried out. It was observed that the error rate between it was less than $1 \%$.

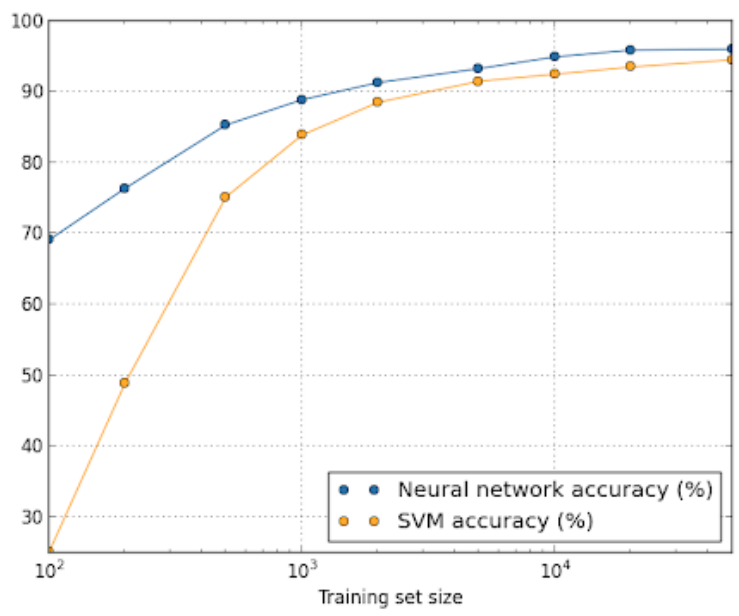

Figure 7. Comparison with SVM
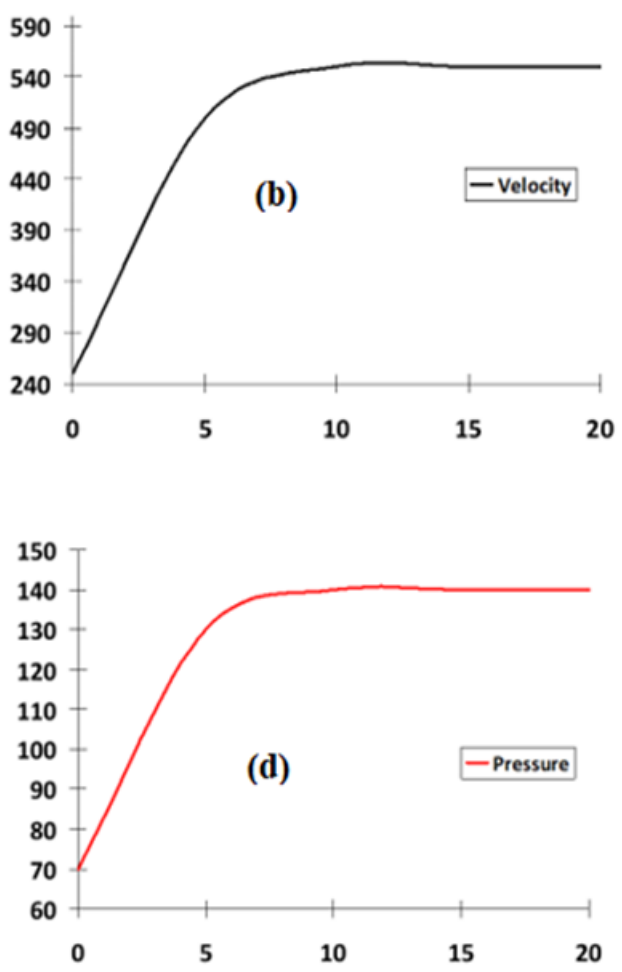

Figure 8. (a) Input variable temperature, (b) Input variable velocity, (c) Input variable humidity, and (d) Input variable pressure 


\section{CONCLUSIONS}

Artificial neural network is capable to solve complex problems by using multiple hidden layers. In this experiment temperature, pressure, velocity and humidity is provided as an input variable to the artificial neural network. Artificial neural network provides an information related to the relationship of input and output variables of the steam turbine. This information is useful for designer while designing the controller of the power plant. Artificial neural network training is based on the input values and the count of epochs configured in the system.

For time optimization a NARMA uses minimum and maximum normalization method in the training phase. In the proposed model for every signal, neural network is created and then a centralised processing unit is used to analyse the overall output of each processing. This reduces the time required for processing and improves the time complexity of the system. Backpropagation technique is used in artificial neural network to feed the error rate between hidden layers, which improves the overall performance of the system. The proposed system maintains the ideal values of the pressure, temperature, velocity and humidity.

\section{REFERENCES}

[1] Li, L., Xu, J.Q., Li, G., Zhou, K.Y., Shi, Y.F. (2011). Optimization research on nozzle governing 600MW steam turbine operation. In 2011 Asia-Pacific Power and Energy Engineering Conference, Wuhan, China, pp. 1-4. https://doi.org/10.1109/APPEEC.2011.5748872

[2] Nowak, G., Rusin, A. (2016). Using the artificial neural network to control the steam turbine heating process. Applied Thermal Engineering, 108: 204-210. https://doi.org/10.1016/j.applthermaleng.2016.07.129

[3] Salim, H., Faisal, K., Jawad, R. (2018). Enhancement of performance for steam turbine in thermal power plants using artificial neural network and electric circuit design. Applied Computational Intelligence and Soft Computing, 2018: 8042498. https://doi.org/10.1155/2018/8042498

[4] Burhani, A., Rozaq, F., Supriyanto, E. (2020). Case study application of cold peening in combination with thermal stress relieving as effective method for straightening large turbine rotor. In 2020 International Conference on Power, Energy, Control and Transmission Systems (ICPECTS), Chennai, India, pp. 1-6. https://doi.org/10.1109/ICPECTS49113.2020.9336994

[5] Dominiczak, K., Rzadkowski, R., Radulski, W. (2016). Temperature and stress steam turbine rotor control based on neural network. Journal of Vibration Engineering \& Technologies, 4(4): 319-326.

[6] Dettori, S., Maddaloni, A., Galli, F., Colla, V., Bucciarelli, F., Checcacci, D., Signorini, A. (2021). Steam turbine rotor stress control through nonlinear model predictive control. Energies, 14(13): 3998. https://doi.org/10.3390/en14133998

[7] Chan, Y.K., Gu, J.C. (2012). Modeling of turbine cycles using a neuro-fuzzy based approach to predict turbine- generator output for nuclear power plants. Energies, 5(1): 101-118. https://doi.org/10.3390/en5010101

[8] Kamble, R.A. (2017). Short and long term stock trend prediction using decision tree. In 2017 International Conference on Intelligent Computing and Control Systems (ICICCS), pp. 1371-1375. https://doi.org/10.1109/ICCONS.2017.8250694

[9] Xin, R., Zhang, J., Shao, Y. (2020). Complex network classification with convolutional neural network. Tsinghua Science and Technology, 25(4): 447-457. https://doi.org/10.26599/TST.2019.9010055

[10] Nezamoddini, N., Gholami, A. (2019). Integrated genetic algorithm and artificial neural network. In 2019 IEEE International Conference on Computational Science and Engineering (CSE) and IEEE International Conference on Embedded and Ubiquitous Computing (EUC), pp. 260-262. https://doi.org/10.1109/CSE/EUC.2019.00057

[11] Khandelwal, A., Kumar, J. (2019). Applications of AI for power electronics and drives systems: A review. In 2019 Innovations in Power and Advanced Computing Technologies (i-PACT), Vellore, India, pp. 1-6. https://doi.org/10.1109/i-PACT44901.2019.8960123

[12] Bajya, R.R., Taparia, M.R. (2016). Modeling of a nonlinear hydro power plant and analysis with PID controllers. In 2016 International Conference on Control, Computing, Communication and Materials (ICCCCM), Allahbad, India, pp. 1-3. https://doi.org/10.1109/ICCCCM.2016.7918262

[13] Kapetanakis, T.N., Vardiambasis, I.O., Lourakis, E.I., Maras, A. (2018). Applying neuro-fuzzy soft computing techniques to the circular loop antenna radiation problem. IEEE Antennas and Wireless Propagation Letters, 17(9): 1673-1676. https://doi.org/10.1109/LAWP.2018.2862939

[14] Nezamoddini, N., Gholami, A. (2019). Integrated genetic algorithm and artificial neural network. In 2019 IEEE International Conference on Computational Science and Engineering (CSE) and IEEE International Conference on Embedded and Ubiquitous Computing (EUC), pp. 260-262. https://doi.org/10.1109/CSE/EUC.2019.00057

[15] Krzywanski, J., Fan, H., Feng, Y., Shaikh, A.R., Fang, M., Wang, Q. (2018). Genetic algorithms and neural networks in optimization of sorbent enhanced $\mathrm{H}_{2}$ production in FB and CFB gasifiers. Energy Conversion and Management, 171: 1651-1661. https://doi.org/10.1016/j.enconman.2018.06.098

[16] Deshpande, P., Warke, N., Khandare, P., Deshpande, V. (2012). Thermal power plant analysis using artificial neural network. In 2012 Nirma University International Conference on Engineering (NUiCONE), Ahmedabad, India, pp. 1-6. https://doi.org/10.1109/NUICONE.2012.6493290

[17] Sadough Vanini, Z.N., Meskin, N., Khorasani, K. (2014). Multiple-model sensor and components fault diagnosis in gas turbine engines using autoassociative neural networks. Journal of Engineering for Gas Turbines and Power, 136(9): https://doi.org/10.1115/1.4027215 